\title{
Investigating the small lung: which imaging procedure?
}

\author{
I GORDON AND P HELMS
}

Department of Paediatric Radiology and Respiratory Unit, Hospital for Sick Children, London

SUMMARY Ventilation and perfusion radionuclide lung scans, using krypton $81 \mathrm{~m}$ and technetium-99m macroaggregates, were performed together with a variety of other imaging procedures in 18 children aged between 1 week and 13 years in whom radiology had shown a small lung. Radionuclide scans provided an assessment of regional ventilation and perfusion unobtainable by other means, and 4 main categories of disturbed function could be seen in the radiological small lung-namely, absence of ventilation and perfusion, absent perfusion with preserved ventilation, generalised or segmental decreases in ventilation, and perfusion and segmental perfusion defects in areas of decreased ventilation. The clinical history and other imaging procedures, including fluoroscopy and penetrated mediastinal views, enabled a firm diagnosis to be made in each of these 18 patients. In 3 main pulmonary arteries were absent, 2 had lung aplasia, 2 had lobar aplasia, 9 had varying degrees of pulmonary hypoplasia (two with additional sequestrated segments), 1 had lobar emphysema, and 1 had post-infective lung mal-development (MacLeod's syndrome).

The child with a radiological small lung poses two main questions for the clinician. Is this finding a manifestation of a congenital abnormality, or has it been acquired? Is there any treatment which could be beneficial and allow for a better functional result as lung growth and development progress? The range of possible imaging procedures is wide, none is without risk, and the risks relating to radiation dosage must be considered. The aim is to avoid over investigating the child but at the same time maintain a high degree of confidence in explaining the radiological appearances. The purpose of this study was to assess the role of $81 \mathrm{~m}$ krypton $(\mathrm{Kr})$ ventilation $(\dot{\mathrm{V}})$ and technetium-99m $\left({ }^{99 \mathrm{~m}} \mathrm{Tc}\right)$ microsphere perfusion (Q) lung scans in such circumstances. There is little discomfort for the child, a lower radiation dose is used than in other second-line imaging procedures, and the investigation can be performed without co-operation from the patient, so making it suitable for young children. ${ }^{1-2}$

\section{Patients and methods}

Eighteen children aged between 1 week and 13 years with unilateral lung disease of unknown aetiology had $\mathrm{Kr}, \dot{\mathrm{V}}$, and ${ }^{99 \mathrm{~m}} \mathrm{Tc} \dot{Q}$ lung scans as part of a variety of other imaging procedures which included fluoroscopy, bronchography, and angiography
(Table). The results of the $\dot{V} / \dot{Q}$ scans and other imaging investigations were discussed at regular clinico-radiological meetings with the clinicians responsible for the children's management, at which time decisions about further investigation and management were also made. Fluoroscopy was carried out in the supine position with a variable $\mathrm{kV}$ using an image intensifier.

\section{Results}

The disturbances found on $\dot{V} / Q$ scanning could be placed in 4 categories-namely complete absence of ventilation and perfusion in one lung, complete absence of perfusion with preserved ventilation in one lung, decreases both of ventilation and perfusion in one lung either generalised or segmental in nature, and segmental perfusion defects in hypoventilated lungs. In each case a diagnosis could be made from the clinical history together with the imaging results. Cases 1,2 , and 3 were shown to have absent main pulmonary arteries, Cases 4 and 5 had total lung aplasia, Cases 6 and 7 had lobar aplasia, Cases 8-16 had varying degrees of pulmonary hypoplasia (two with additional sequestrated segments), Case 17 had lobar emphysema, and Case 18 had predominantly unilateral post-infective lung mal-development (MacLeod's syndrome) ${ }^{3}$ (Table). 
Table Details of patients

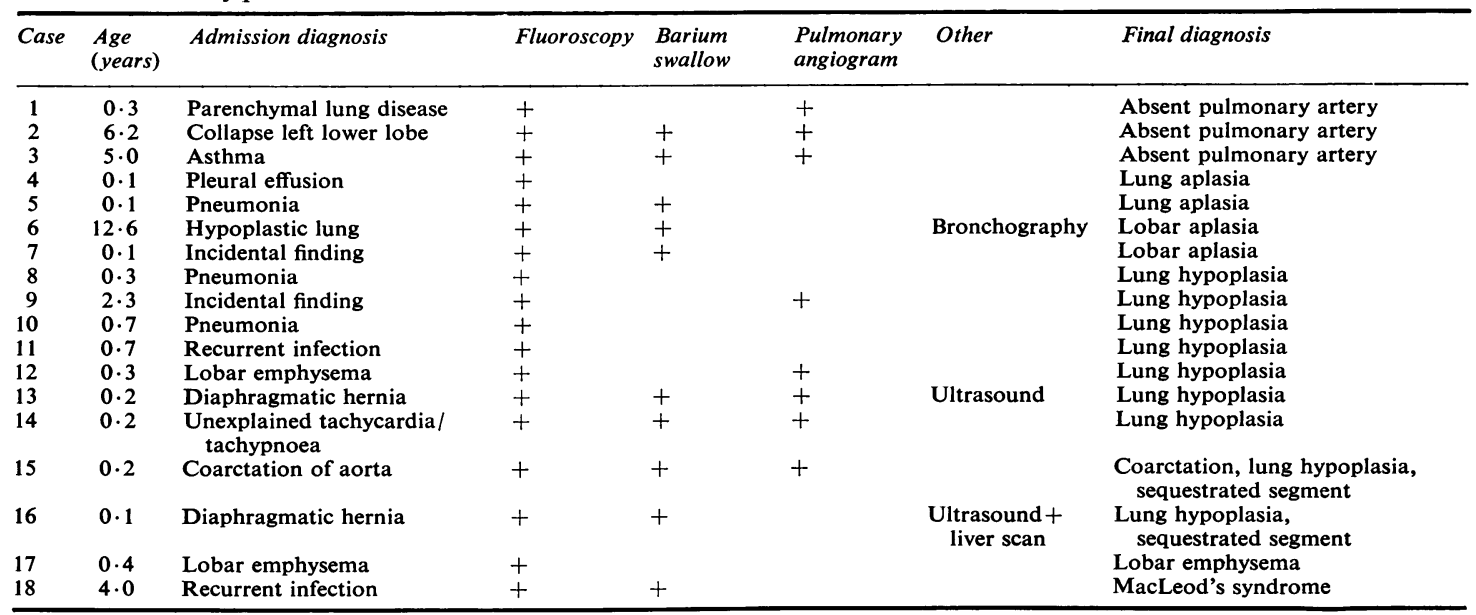

+ Examination carried out.

Complete absence of $\dot{\mathbf{V}} / \dot{\mathbf{Q}}$ in one lung. Three children were found to have a non-functioning lung on $\dot{V} / \dot{Q}$ scanning. Chest radiographs in 2 of them showed opaque hemithoraces with mediastinal shift to the affected side strongly suggesting a diagnosis of pulmonary aplasia. This diagnosis was established when penetrated mediastinal radiographs showed absent main stem bronchi on the affected side. The remaining child had an aerated small lung on chest radiography, and fluoroscopy showed paradoxical movement of a raised hemidiaphragm. A main bronchus was also seen and a diagnosis of severe pulmonary hypoplasia was made (Fig. 1).

Complete absence of $\dot{\mathbf{Q}}$ in a ventilated lung. Three children showed decreased $\dot{V}$ on the side of the small lung with total absence of $\dot{Q}$. The chest radiographs showed small lungs with mediastinal displacement to the affected side. This pattern was caused by a congenital absence of the pulmonary artery and was confirmed at pulmonary angiography in each case (Fig. 2).

Decreased $\dot{\mathbf{V}} / \dot{\mathbf{Q}}$ in one lung. In 13 children pronounced decreases in both $\dot{V}$ and $\dot{Q}$ were observed in one lung. The chest radiographs showed small hemithoraces containing aerated lung with mediastinal displacement to the affected side (Fig. 3 ). These abnormalities were present in 9 of the 13 children. $\dot{\mathrm{V}} / \mathbf{Q}$ scans showed loss of the normal anatomical landmarks on the oblique projections which suggested a diagnosis of pulmonary hypoplasia. One child was found to have air trapping on the affected side during fluoroscopy with patchy areas of poor $\dot{V}$ and $\dot{Q}$ in the opposite lung. These findings, together with a history of severe lower respiratory tract infection in early childhood, suggested the diagnosis of unilateral post-infective lung mal-development (MacLeod's syndrome). ${ }^{3}$

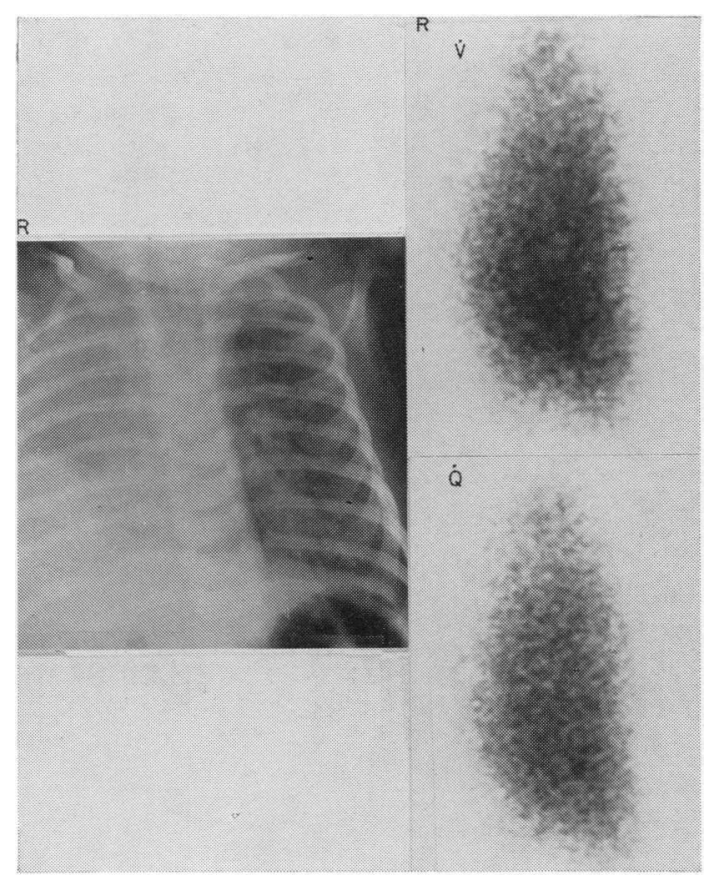

Fig. 1 Chest radiograph shows mediastinal displacement to the right with a raised right hemidiaphragm. Aerated right lung is noted in the small hemithorax. Radionuclide scan demonstrates homogeneous $\dot{V} / \dot{Q}$ in the left lung with virtually no activity in the right. 


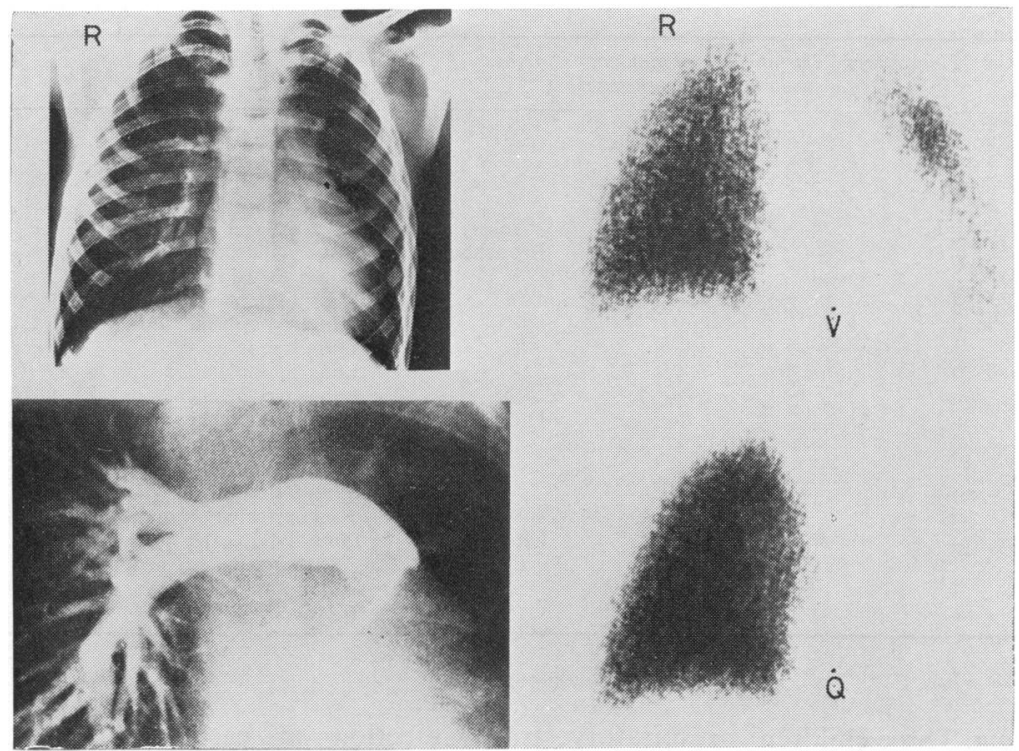

Fig. 2 Chest radiograph shows a small left hemithorax containing a small hypertransradiant left lung. The $\dot{V}$ scan shows decreased overall ventilation of the left lung while the $\dot{Q}$ scan shows absence of perfusion; the right lung appears to be normally ventilated and perfused. The pulmonary angiogram showed an absent left pulmonary artery.
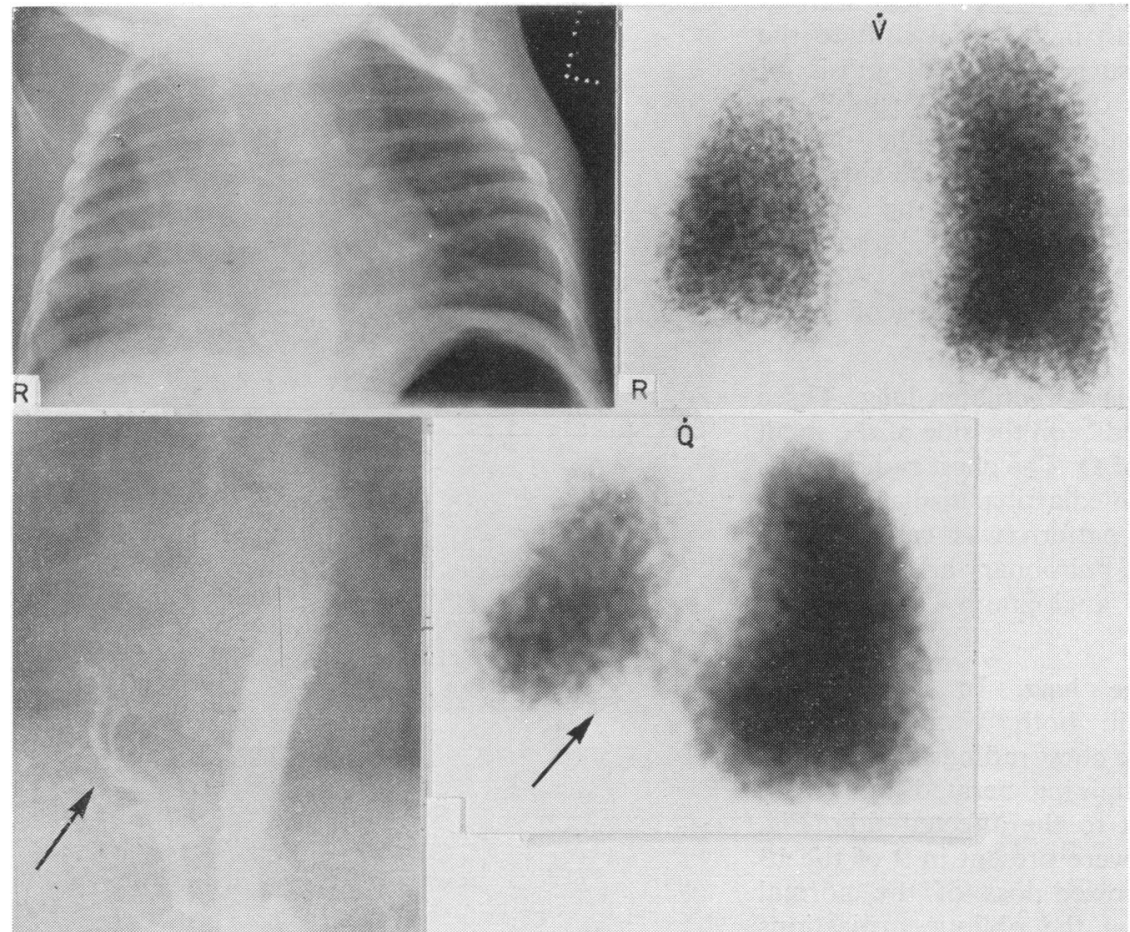

Fig. 3 Chest radiograph shows a small right lung with mediastinal displacement to the right; the hemidiaphragms appear to be normal. The $\dot{V}$ scan shows a well ventilated left lung with overall poor ventilation of the right. The $\dot{Q}$ scan shows a poorly perfused right lung with a segmental defect at the base medially (arrow). Aortogram shows systemic supply to the non-perfused area at the right base (arrow) confirming the diagnosis of a sequestrated segment in the hypoplastic right lung. There is an increased incidence of sequestrated segments in hypoplastic lungs. 
In 2 of the 3 remaining children the decreased $\dot{V} / \dot{Q}$ was noted to be mainly in the region of the upper lobe suggesting aplasia of a single lobe. One of these children had severe exercise limitation with marked expiratory airflow obstruction not reversible with bronchodilators. Because of this finding and the limited abnormality demonstrated on $\dot{V} / \dot{Q}$ scanning, bronchoscopy and bronchography were performed. These studies demonstrated a fixed stenosis of the lower trachea, complete absence of a right upper lobe bronchus, and a long segment tracheomalacia in the left main bronchus (Fig. 4). The remaining child had a segmental $\dot{V} / \mathbf{Q}$ defect in the region of the lingula. Fluoroscopy revealed a fixed hyperinflated area in the left lung with air trapping, the chest radiograph showed mediastinal displacement to the right; lobar emphysema was diagnosed and confirmed at thoracotomy.

\section{Segmental $\dot{Q}$ defect in a hypoventilated lung. A single}

segmental Q defect was noted in 2 of the 9 children with a small hypoventilated lung, raising the additional differential diagnosis of pulmonary emboli or sequestration in the affected lungs. Sequestration on the affected lung was confirmed in both cases, one at surgery, the other on aortography (Fig. 3).

\section{Discussion}

In all these children the $\dot{V} / \dot{Q}$ scans provided valuable information on the site and extent of the abnormalities of ventilation and perfusion in the lungs, and in 2 children with pulmonary hypoplasia the scans led to the additional diagnosis of sequestration. The importance of taking a clinical history and any relevant functional information into account was demonstrated in the child (Case 6) who was shown to have a more complex pattern of lung disease than had immediately been apparent (Fig. 4).

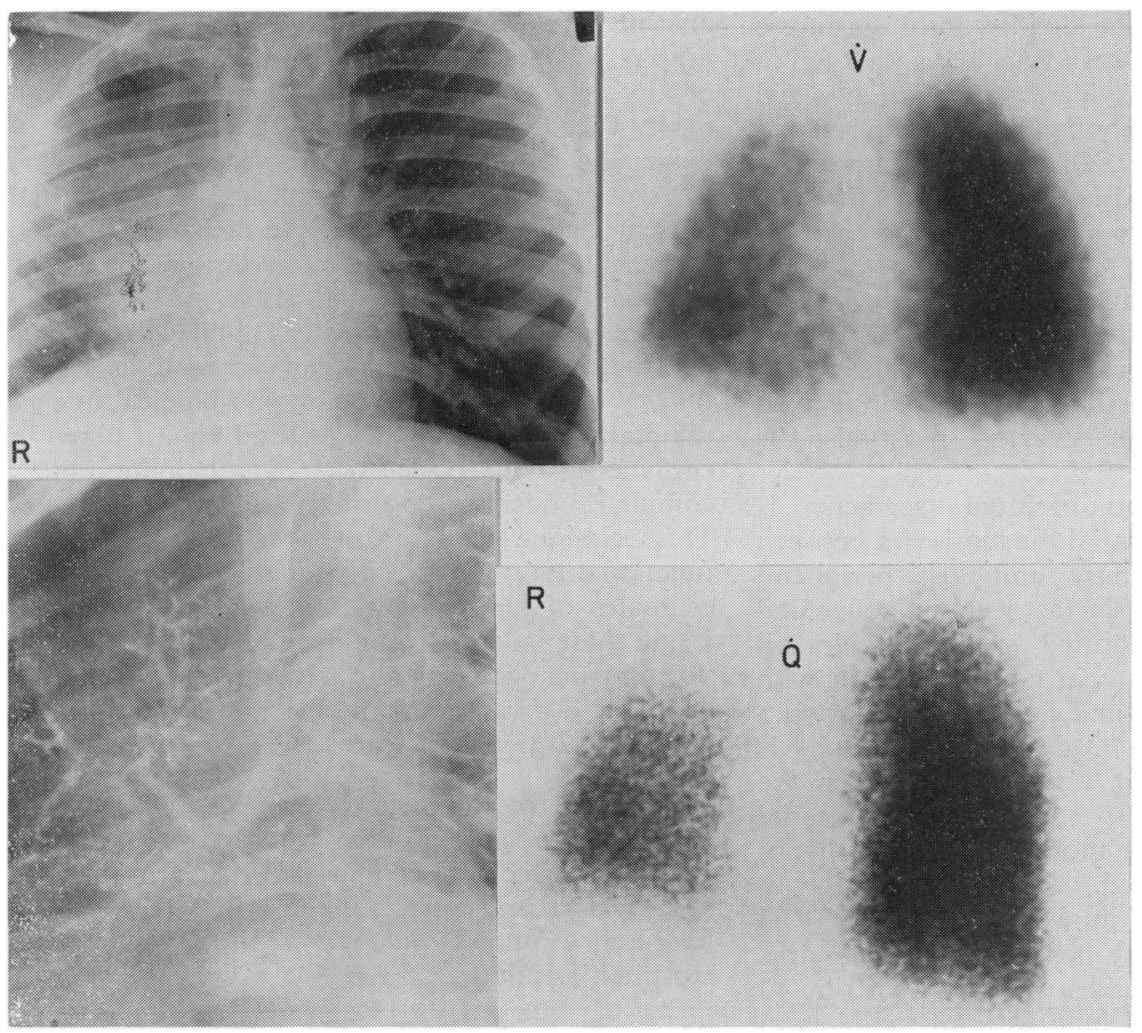

Fig. 4 (Case 6.) Chest radiograph shows abnormalities of the posterior aspect of the right 5th and 6th ribs with the mediastinum shifted to the right; a small right lung can also be seen. The $\dot{V}$ scan shows a well ventilated left lung and a poorly ventilated right. The $\dot{Q}$ scan is similar in appearance to the $\dot{V}$ scan. Bronchogram demonstrates an absent right upper lobe bronchus confirming the diagnosis of a congenital absence of the right upper lobe. The narrowing of the lower end of the trachea can also been seen. 
In children with only one functioning lung the differential diagnosis must include collapse/consolidation, aplasia, or severe hypoplasia. In such cases penetrated views of the mediastinum in order to visualise the main stem bronchi, and chest fluoroscopy to assess the ventilatory function were invaluable. If no main bronchi or lung movement could be detected the diagnosis of lung aplasia was made. If bronchi could be seen but no aerated lung observed, a diagnosis of collapse or consolidation was made. If collapse or consolidation is present, $\mathbf{Q}$ in the affected area may be partially preserved although $\dot{V}$ is absent (unpublished observations). In the 9 children with unilateral pulmonary hypoplasia, fluoroscopy showed a small aerated segment of lung on the affected side.

In adults, pulmonary emboli or bronchial carcinoma are the most important causes of pure perfusion abnormalities. In young children however, the absence of perfusion is virtually diagnostic of a congenital absence of the pulmonary artery on the affected side. This can be divided into 3 groups ${ }^{4}$ : absent left pulmonary artery associated with a Fallot tetralogy and increased incidence of right-sided aortic arch, ${ }^{5}$ extracardiac anomalies, ${ }^{6}$ and an isolated absent pulmonary artery. Of the last group about half are on the right and half on the left. ${ }^{7}$ In such cases the $\dot{V} / \dot{Q}$ scan is diagnostic, and cardiac catheterisation may be carried out to evaluate pulmonary arterial pressure since pulmonary hypertension is liable to develop. If the chest radiograph shows a large hypertransradiant lung on the side of the absent $Q$ then the possibility of an extrinsic mass pressing on the pulmonary artery must also be considered. ${ }^{8}$

If a ventilated non-perfused segment is demonstrated the differential diagnosis lies between a single small pulmonary embolus, peripheral pulmonary artery stenosis (as seen in congenital rubella), or a sequestrated segment. Not all sequestrated segments are in communication with the bronchial tree as in our 2 cases, ${ }^{9}$ so the lung scan may show a matched $\dot{V} / Q$ defect. Aortography is not only diagnostic but also important for the surgeon to establish the source of the arterial supply. Sequestration is more common in hypoplastic lungs, as illustrated.

If the affected lung showed pronounced $\dot{\mathrm{V}} / \dot{Q}$ abnormalities, lack of the normal lobar landmarks on the oblique views, and a normally distributed $\dot{V} / \dot{Q}$ in the contralateral lung without radiological evidence of acute lobar collapse/consolidation, the diagnosis of pulmonary hypoplasia could be made. This pattern of abnormality has also been found in infants studied after successful repair of congenital diaphragmatic hernia on whom longitudinal studies of lung function and $\dot{V} / Q$ scans are in progress (unpublished observations). It was also possible in one child to demonstrate a congenital absence of a lobar segment. In the single child with acquired disease (MacLeod's syndrome) ${ }^{3}$ abnormalities were also noted on the $\dot{V} / Q$ scan in the apparently radiologically normal lung which would perhaps be expected as a more likely outcome of severe pulmonary infection than a complete sparing of one lung. McKenzie et al.$^{10}$ using a single radionuclide $13 \mathrm{~N}$ and a 4 zone regional washout technique, together with other imaging techniques, found MacLeod's syndrome to be a rare cause of unilateral pulmonary hypertransradiancy and argued the case in favour of investigating the child with a radiological hyperlucent hemithorax.

We deliberately chose a descriptive approach to our $\dot{V} / \dot{Q}$ scans with multiple views around the chest in order that the abnormalities could be localised at lobar, segmental, or subsegmental level. This approach enables a direct comparison with the anatomical information provided on chest radiography. $\dot{V} / Q$ lung scans can be performed in children of any age; the radiation dose is lower than with most other imaging techniques ${ }^{1}$ and a high level of diagnostic certainty can be achieved without having to resort to more invasive methods. Most regional nuclear medical centres have the necessary krypton 81 generators. The ability to focus attention on the essential functional abnormalities suggests that $\dot{V} / \dot{Q}$ lung scans should be more widely applied in children and that they should be an early imaging investigation. $\dot{V} / \dot{Q}$ scans provide pathophysiological images unobtainable by any other method and, if taken in conjunction with the chest radiograph, allow a more complete understanding of the underlying disease. This technique also offers the possibility of following functional lung growth in young children in whom estimates of lung function are difficult to obtain. Using these imaging techniques cardiac catheterisation and angiography are not required for diagnostic purposes but rather to outline the anatomy more clearly if surgery is indicated.

We thank the physicians and surgeons at The Hospital for Sick Children who referred patients, and S E Coote for secretarial assistance. Krypton $81 \mathrm{~m}$ generators were supplied by the Medical Research Council's cyclotron at the Hammersmith Hospital, London.

P H was supported by the Medical Research Council.

\section{References}

1 Gordon I, Helms P, Fazio F. Clinical applications of radionuclide lung scanning in infants and children. $B r J$ Radiol 1981 ; 54 : 576-85. 
${ }^{2}$ Li D K, Treves S, Heyman S, Kirkpatrick J A, Jr, Lambrecht R M, Ruth T J, Wolf A P. Krypton $81 \mathrm{~m}$ : a better radiopharmaceutical for assessment of regional lung function in children. Radiology 1979; 130: 741-7.

3 MacLeod W M. Abnormal transradiancy of one lung. Thorax 1954; 9: 147-53.

4 Rees S. Arterial connections of the lung. Clin Radiol 1981; 32: 1-15.

5 Emanuel $\mathbf{R}$ W, Pattinson $J$ N. Absence of the left pulmonary artery in Fallot's tetraiogy. Br Heart $J$ 1956; 18: 289-95.

6 Pool P E, Vogel J H K, Blount S G, Jr. Congenital unilateral absence of a pulmonary artery. The importance of flow in pulmonary hypertension. Am J Cardiol 1962; 10: 706-32.

7 Shakibi J G, Rastan H, Nazarian I, Paydar M, Aryanpour I, Siassi B. Isolated unilateral absence of the pulmonary artery. Review of the world literature and guidelines for surgical repair. Jpn Heart $J$ 1978; 19 : 439-51.

8 Alderson P O, Gilday D L, Wagner H N, Jr. In: Atlas of pediatric nuclear medicine. St Louis: Mosby, 1978: 94-5.

- Buntain W L, Woolley M M, Mahour G H, Isaacs H, Jr, Payne V, Jr. Pulmonary sequestration in children: a twenty five year experience. Surgery $1977 ; 81$ : 413-20.

10 McKenzie S A, Allison D J, Singh M P, Godfrey S. Unilateral hyperlucent lung. The case for investigation. Thorax 1980; 35: 745-50.

Correspondence to Dr I Gordon, Department of Paediatric Radiology, Hospital for Sick Children, Great Ormond Street, London WC1N 3JH.

Received 16 February 1982

\section{British Paediatric Association}

Annual meetings

1983 12-16 April York University

1984 10-14 April York University

1985 16-20 April York University

1986 15-19 April York University 